\title{
An algorithm for precise localization of measurements in rolling stock-based diagnostic
}

systems

\author{
M. Carnevale ${ }^{1}$, I. La Paglia ${ }^{2}$, P. Pennacchi ${ }^{2}$ \\ ${ }^{1}$ Università degli Studi di Pavia, Dipartimento di Ingegneria Industriale e dell'Informazione, \\ Via A.Ferrata 5, 27100 Pavia, Italy; \\ 2 Politecnico di Milano, Dipartimento di Meccanica, \\ Via La Masa 1, 20156 Milano, Italy \\ Corresponding author: marco.carnevale@unipv.it
}

\section{Post-print version}

To cite this paper:

Carnevale, M., La Paglia, I., Pennacchi, P. An algorithm for precise localization of measurements in rolling stock-based diagnostic systems (2020) Proceedings of the Institution of Mechanical Engineers, Part F: Journal of Rail and Rapid Transit, DOI: 10.1177/0954409720965798

Editor version: https://journals.sagepub.com/doi/full/10.1177/0954409720965798

\begin{abstract}
The paper describes the development and the validation of an algorithm for the geo-localization of trains, specifically designed for diagnostic applications of rolling stock and infrastructure. The algorithm exploits two signals commonly available on commercial trains, namely odometry and GPS, combined to overcome the drawbacks they both show when adopted individually. The algorithm's flow consists of a map matching procedure for the projection of GPS acquisitions on digital maps of the railway line, followed by a robust fit to correlate the map-matched data to the odometry data. The system is specifically conceived to be adopted in rolling-stock based diagnostic system, to correlate the diagnostic indices to the actual position where they are gathered along the line. It is specifically designed to operate on double-track railways, even if its adoption in multi-track scenario typical of commuter rail would be possible by means of further integration with balise signals or other train control data.

Results from track tests on a high-speed train with instrumented pantographs and bogies show that the developed algorithm allows to obtain a good repeatability of diagnostic indicators collected during repeated runs of the train along the same track. This result set the basis for the automation of data analysis in a wayside server, and for the computerization of the comparison of data belonging to homogeneous track sections, so as to perform a trend analysis.
\end{abstract}

Keywords: Condition based maintenance; rolling stock based diagnostic systems; train geo-localization. 


\section{Introduction}

Traffic growth in rail transportation poses stricter requirements on the reliability and availability of the railway system, so that Condition Monitoring $(\mathrm{CM})$ and Condition Based Maintenance (CBM) of both railway vehicles and infrastructure are one of the new challenges in the railway industry [1]. Up until recently, infrastructure managers and railway operators have been relying on planned inspection and time-based or distance-based preventive maintenance, which is not always a fault-proof nor cost effective method. In the case of pantographs, as an example, a visual inspection of the contact strip wear is carried out periodically (e.g. monthly) by a team of workers inspecting the vehicle, and both the strips are replaced if at least one requires to be substituted. Other examples are the check of the upward force, that is a fundamental parameter for pantograph operation, or the check of dampers at the base of the articulated frame. These checks are also carried out periodically, usually every year or after some hundreds of thousands of kilometres. Nevertheless, visual inspection and planned preventive maintenance are not effective in providing continuous information about the actual condition of the components, making it hard to identify incipient faults before that a damage, or worse an accident, occurs.

Previous research works on the topic have outlined that a rolling stock-based solution [2] with acceleration sensors installed on-board train [3],[4],[5] would be more effective and money-saving than infrastructure based solutions, allowing to monitor at the same time both the status of the infrastructure (track and overhead contact line), and of rolling stock components (bogies or pantographs). When an entire train fleet is equipped with simple sensor systems and data are analysed in real-time with suitable algorithms running on-board train, performance indices like RMS [4],[5] can be transmitted and stored in a ground server for a trend analysis. The presence of anomalous signals in the data coming from a single train would indicate a malfunctioning of a rolling stock component, whereas anomalous data acquired at a certain position of the line from most of the trains in the fleet would identify a defect in the infrastructure. 
The capability to associate diagnostic indicators to the actual position where they are gathered along the track is essential for the purpose of condition monitoring, regardless of whether data are aimed at rolling stock or infrastructure monitoring. If in the case of infrastructure monitoring the benefit resulting from the possibility to send workers at the exact location of the damage is easy to understand, the need for accurate location might be not intuitively caught when considering the monitoring of rolling stock components: the power content of bogie, car-body, axle-box and even pantographs accelerations acquired on-board train can be relevantly dependent on the track section due to differences in the quality of the infrastructure, the latter depending on different traffic loads, different geographical areas, or even different working accuracy of the maintenance teams. As a consequence, for a given train type, the collected data can show relevant differences even at a given speed and for given nominal design and parameters of the infrastructure, so that it is not possible to set a general threshold value only dependent on speed and nominal track. Trend analysis of diagnostic indicators should be carried out on the contrary for well-defined and repeatable track sections, so that incipient damages can be identified searching for trend variations in data corresponding to a specific position along the line.

Current technology and state of the art in the European Train Control System (ETCS) relies on the signal transmitted by eurobalises placed along the rails and univocally codified, so that the exact position of the train is available both on-board and in the ground control room. However, difficulties may arise when trying to adopt the signal received from eurobalises for the purposes of a condition monitoring system [6]: from a technical point of view, the synchronization of the positioning data from balises and acceleration data gathered on-board train would not be straightforward, especially when the condition monitoring system has to be installed as a retrofit on already certified vehicles. A CBM system linked to the main vehicle logic to sniff balises' signals would have indeed an impact on the risk analysis of the entire system. Even when accepted from Notified Bodies and National Railways Safety Agencies, this would still require the repetition of the certification process, with the consequent high costs involved. 
For these reasons, it is authors' opinion that a suitable path to be followed consists in a stand-alone solution which should be able to identify the actual milestone position of the train along the track and to associate it to diagnostic indicators generated from the sensors installed on the train. On the other hand, when operating in a multi-track environment, the adoption of balise signals or other train control data could be necessary to determine which track the rolling-stock is running on, standard GPS accuracy being not suitable for the purpose.

In the aforementioned scenario several works have been proposed in the literature dealing with the issue of geo-localization of a train and with the necessity to link acceleration data measured on board to their actual mileage position along the track. The need of a precise and standardised procedure for generating digital map of the railway network is recognized in [7]: to cope with the lack of accuracy of GNSS data, map-matching techniques allows to match the trajectory followed by a vehicle to a digital map of the railway network, and to correlate the data provided by different positioning sensors to the same digital map. In [8] the authors present an algorithm for automatic track identification, able to recognize the path followed by a rail vehicle. The algorithm, based on the Least Trimmed Square Hausdorff Distance (LTS-HD), relies on a precise digital map and GNSS information alone. The track on which the vehicle is running is identified by comparing the incoming position samples with the digital map, to find the best match, the major limitation being the non-continuous availability of GSNN data.

The possibility to exploit other instruments than GPS in map-matching procedures has been investigated by correlating the track curvature from the digital map to the corresponding measurements of a yaw gyroscope and a tachometer located on board train in [9] and [10], and by fusing the data of a GPS receiver with radar and inertial sensors in [11] and [12].

Sensor fusion between tachometer and Inertial Measurements Unit (IMU) has been proposed to improve the accuracy achievable through the mere odometry in train localization applications related to safety issues [13]. However, applications for condition monitoring can in general admit a lower accuracy, in the order of some tens of meters. 
The works in [14],[15],[16] describe a system to identify track geometry irregularities (vertical and lateral components) by means of carbody mounted accelerometers installed on board of an in-service vehicle. The proposed approach, validated against dedicated track recording vehicle, relies on a GPS system and map-matching algorithm to tag the location of faults in track. The latter are identified as the events in which accelerations or estimated track irregularities exceed proper threshold levels. Finally, an example of successful condition monitoring systems installed on in-service Acela trains (USA) is presented in [17]. Trains are equipped with a remotely controlled monitoring system which provides continuous measurements of vehicle motion, detects various acceleration events and tag them with GPS information. The GPS module used to tag data with time and location showed an accuracy of 60-90 m.

This paper describes a positioning system able to correlate diagnostic indicators to the actual track section where they are gathered. The system has been designed in the framework of a more general project for the development of a rolling stock-based condition monitoring system, in which bogie and pantograph accelerations are acquired and processed in real time on-board train to obtain diagnostic indicators, which are then transmitted to a ground server [4]. The aim of the work is the development of an algorithm which enables the possibility to overlap and to automatically compare the signals from several train runs on the same track, with a target accuracy of some tens of meters (i.e. maximum error around $20 \mathrm{~m}$ ). The complete system has been firstly tested on a in-service commuter train [3], and then adapted and extended to high-speed applications. It is autonomous in terms of hardware and software, and therefore can be retrofitted on existing trains. It is specifically thought to operate on double-track scenarios typical of high-speed lines, and its adoption in multi-track environment would require further integration with balise signals or other operational and train control data, to identify the track along which the rolling-stock is running.

The paper is organized as follows: section 2 describes the proposed hardware and its integration with the diagnostic units for pantograph and bogie signal processing. Section 3 illustrates the principles of the algorithm, which is composed of two steps consisting in a map matching procedure and in the 
application of a robust fit method, using M-estimators [18]. Section 4 presents the experimental validation obtained through the implementation of the proposed system on a high-speed train and tests up to $300 \mathrm{~km} / \mathrm{h}$. The achievements in terms of repeatability of the diagnostic indicators for several runs of the train on the same track are particularly emphasized, being the pre-requisite for the automation of data analysis in a ground server and for the development of a procedure for conditionbased maintenance. Finally, conclusions are drawn in section 5.

\section{System layout and integration with diagnostic units}

The diagnostic system conceived and designed in the last decade at the Department of Mechanical Engineering of Politecnico di Milano [3],[4],[5],[6] is based on electronic boards for the acquisition of acceleration data from bogies and pantographs, and for their processing through suitable diagnostic algorithms. The outputs, evaluated in real time during the train run, are synthetic indicators of the status of both the rolling stock components (bogies and pantographs) and of the infrastructure (track and overhead contact line). As an example, bogie and pantograph electronic boards evaluate RMS of accelerations over proper time-based or space-based windows, with a proper level of overlap $[3],[4],[5]$. These data need to be correlated to the actual position where they are gathered along the line, before being sent to a wayside server in which they can be post processed for trend analysis. In order to be suitable for diagnostic applications in commercial trains, the positioning algorithm should work as a stand-alone system, executable without manual supervision. In addition to accuracy, a relevant requirement consists in the capability to output positioning data with continuity, so that the diagnostic boards can generate their indicators as a function of the position along the line, producing repeatable "signatures" of a specific train on a specific line. The output of the algorithm should enable in such a way the computerized comparison of several acquisitions on the same line.

This target cannot be achieved through the individual use of neither GPS nor odometry signal [19]. The accuracy of a standard GPS signals commonly adopted in railway vehicle (i.e.17 $\mathrm{m}$ [20]) might be acceptable for diagnostic applications, since, when giving indications to maintenance operators, 
this error would not prevent the possibility to identify and repair the defect. The accuracy of standard GPS can be even increased up to $3 \mathrm{~m}$ when an EGNOS system is adopted [20]. However, one main drawback consists in the fact that GPS signal is not available in tunnels and may present outliers which would corrupt the correct localization of the data, and therefore the trend analysis process. Figure 1 reports an example of a typical GPS acquisition from a standard commercial train. The dashed line represents the railway track and the star markers the GPS acquisition. The presence of an outlier can be easily identified.

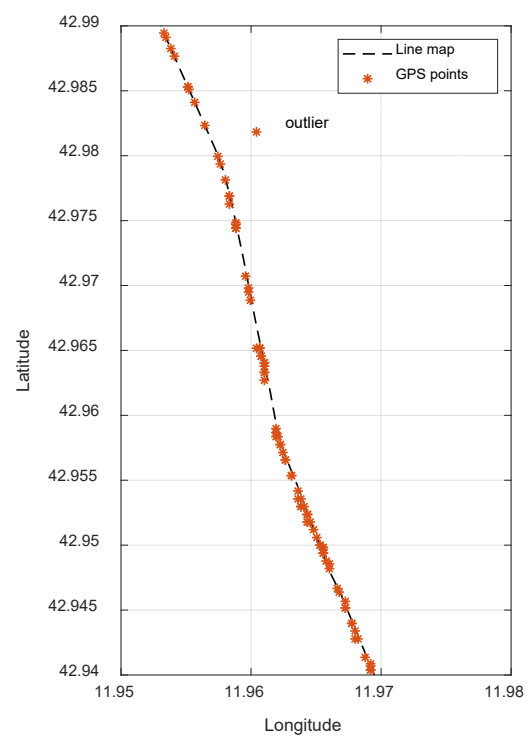

Figure 1: Exemplary GPS acquisitions on a standard train. Presence of outliers.

On the other hand, odometry signal is likely to accumulate errors due to drifts during the train run, especially occurring during acceleration and braking phases. In the former case, due to wheel sleep, the odometry can overestimate the actual distance covered by the train, whereas, during braking, wheel locks may lead to underestimate the covered distance, even if anti-lock systems are effectively working. As an example, Figure 2 a reports a speed profile acquired during a test run on a high-speed train, in which relevant decelerations are visible at $\mathrm{km} 36$ and $\mathrm{km} 79$. The bottom Figure $2 \mathrm{~b}$ reports the angular coefficients of the lines fitting the actual distance covered by the train and the distance evaluated through odometry signal. The angular coefficients of these lines are mainly dependent on the odometry behaviour, since the actual distance covered by the train is evaluated through EGNOS 
GPS system, and therefore regarded as very reliable after post-processing. In the ideal condition, in which the odometry shows no drifts, the angular coefficients should be equal or close to 1 , meaning that the two sensors provide the same output in terms of distance covered by the train. However, during acceleration and deceleration phases the values of the angular coefficients deviate from the unit value, as highlighted in Figure $2 b$.
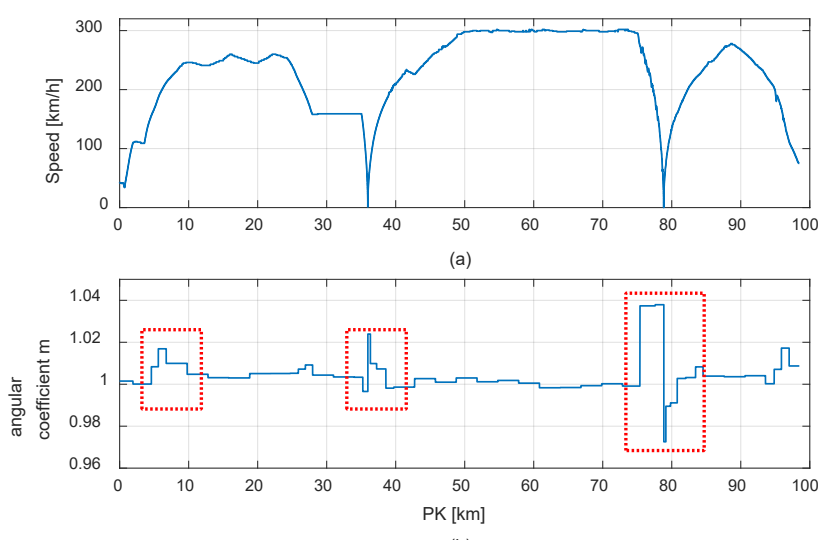

(b)

Figure 2: Exemplary results of drift in the odometry. a) Speed profile of the train run. b) Angular coefficient of the line interpolating the actual distance covered by the train (estimated through EGNOS GPS) and the distance measured by odometry.

For this reason, odometry cannot be adopted on its own to localize diagnostic data along the line.

The geo-localization algorithm described in the present paper tries to integrate the information supplied by GPS data and odometry, to overcome the shortages which they demonstrate when adopted individually. The proposed system relies on the simultaneous acquisition of GPS coordinates and odometry, and on a database containing the digital maps of the railway line in term of latitude, longitude and corresponding milestone position. The latter represents the kilometric position along the railway line, defined by the infrastructure manager and normally adopted to identify the location of all the geometrical characteristics of the infrastructure (e.g. switches, curves, bridges, contact wire changes in overhead contact line, and so on). The railway map exploited in this project represents the midline between two tracks and demonstrated to be sufficient to the aim of this work.

In order to improve the accuracy of the application, the refresh time for GPS coordinates is set equal to $250 \mathrm{~ms}$, which is a lower value compared to the refresh time of $1 \mathrm{~s}$ normally adopted in standard 
railway applications. On the other hand, the odometry signal is sampled during the train run with a time period of $100 \mathrm{~ms}$. These data are set to be used with a high-speed train application, and the requirements might be loosened for lower speeds, like in the case of commuter trains.

The algorithm, described in detail in the following section 3, is run on a dedicated electronic board synchronized with all the other diagnostic systems on bogies and pantographs through proper NTP protocol or similar.

Since pantograph and bogie boards evaluate the RMS based on space windows [3],[4],[5], the geolocalization algorithm triggers the starts and ends of each window based on an output signal sent to the diagnostic boards through Ethernet bus. The triggering function is a very relevant feature, allowing to calculate RMS values always on the same line section (e.g. from starting at $\mathrm{km} 80$ and ending at $\mathrm{km} 80.1$ ), for several passages of each train on the same line and of several trains on the same line. This strongly enables the data repeatability, and the possibility to perform an automatic trend analysis of the data corresponding to a given position of the railway infrastructure. The transmission through Ethernet bus can generate a certain delay from the instant when the position data is generated to the instant in which the trigger gets to diagnostic boards, affecting the start and end position of each window in the order of few meters (at high speed). This delay can be surely tolerated, since the RMS corresponding to each window is not significantly affected by a small difference in the window's start and end.

On the other hand, in order to avoid the effect of delay in the association of diagnostic data to the actual position they refer to, the output of the positioning algorithm is not transmitted directly to pantographs and bogie units, but it is linked to the absolute time corresponding to the instant when GPS and odometry samples have been acquired. At the same time, the output of the diagnostic algorithms of pantograph and bogie boards are linked to the absolute time they have been acquired. Both position and diagnostic data are then transmitted to a central diagnostic unit on-board train and linked, relying on the time vector. Since all the diagnostic boards of the vehicle have synchronous time, this process allows to avoid any latency due to data transmission. 
Data packages containing the diagnostic indicators linked to their actual position are then transmitted to a wayside server, together with the train's identifier and operational info such as train orientation, train speed, operating pantograph. This information is necessary for the automation of the trend analysis process.

\section{Architecture of the positioning algorithm}

The algorithm's input consists in a data cluster $G P S_{i}$ including latitude, longitude and odometry, acquired every $100 \mathrm{~ms}\left(\operatorname{lat}_{i}, \operatorname{long}_{i}, o d_{i}\right.$ at time $\left.t_{i}\right)$. Two parallel tasks run simultaneously: a mapmatching procedure, which projects the acquired GPS data on a digital map of the railway line, and a robust fit algorithm, exploited to find a correlation between the map matched data and the corresponding odometry signal. Since the sampling frequency of the odometry data is necessarily higher than the GPS refresh time, the algorithm can manage the circumstance in which a geodetic coordinate (i.e. latitude and longitude, elevation is neglected) is equal to the previous acquisition: when a new data cluster $G P S_{i}$ is available, a check on preliminary requirements is done, verifying that GPS and odometry signals are valid (i.e. different from zero or corrupted data), and that they differ from the previous acquisition. In such a case, the map matching procedure is applied.

\subsection{Map matching}

This procedure allows to project the GPS acquisitions on a digital map of the railway line, so as to estimate the position of the train along the track. The national railway line is divided in different files, previously stored in the memory of the electronic boards running the algorithm. When the train enters one among the available digital maps, identified by comparison with the actual GPS acquisitions, the current map is automatically uploaded, and the map matching procedure starts. The digital map is a discrete representation of the railway track, composed of a set of points distanced about $1 \mathrm{~km}$ one from each other and defined by latitude, longitude and the corresponding milestone position (i.e. kilometric position). Figure 3 reports a schematic view of the process: the points defining the digital 
map are represented as black stars, and the railway track is approximated with straight lines linking subsequent points (represented as dashed lines).

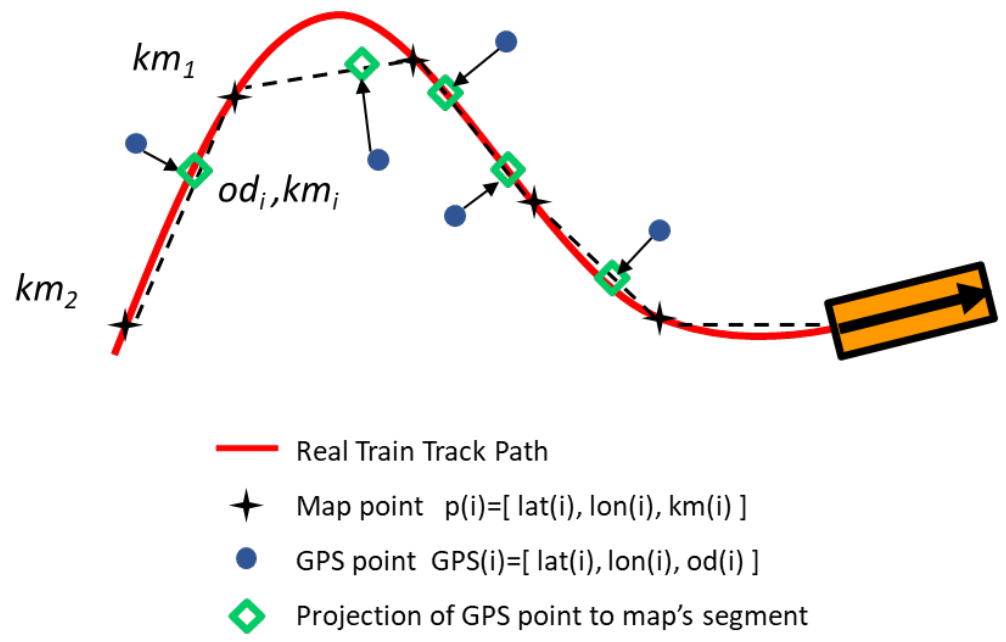

Figure 3: Schematic view of the map-matching procedure.

During the train run, the current GPS acquisitions (represented with filled blue dots in Figure 3) are projected on the dashed lines connecting the points of the digital map, evaluating their geometric distance from the segment. An iterative check in the algorithm allows to identify univocally the segment on which the point must be projected. The projection points are represented with the empty green squares in Figure 3. After this step, the milestone position corresponding to the projected point is estimated based on its distance from the two extremes of the segment under consideration, whose milestone positions are defined in the digital map. An estimated kilometric position $\mathrm{km}_{i}$ is found in such a way. This estimated position is then associated to the odometry acquired at the same time as the original GPS point, so that the output of the map-matching is a set of points $\left(o d_{i}, k m_{i}\right)$ including the estimated milestone position $\mathrm{km}_{i}$ and the corresponding odometry $o d_{i}$.

Due to the standard GPS accuracy, the acquired GPS coordinates do not correspond exactly to the railway track, so that the milestone positions $\mathrm{km}_{i}$ estimated through the map-matching procedure show some irregularities. They cannot be directly adopted for linkage to diagnostic indicators since this would hinder the possibility of getting repeatable signals. Figure 4 shows an example of the output of the map-matching procedure applied to the GPS acquired on the Driver Information System 
(DIS) of a commercial train (maximum speed $250 \mathrm{~km} / \mathrm{h}$ ). The train is equipped with a standard type GPS system, whose typical values of accuracy in locating positional information is in the order $17 \mathrm{~m}$ according to [20]. The couple $\left(o d_{i}, k m_{i}\right)$, resulting from the map-matching procedure as described above, is plotted with the estimated kilometric position $k m_{i}$ on the $y$-axis and the corresponding odometry $o d_{i}$ on the x-axis. The reported data correspond to a track section of $5 \mathrm{~km}$.

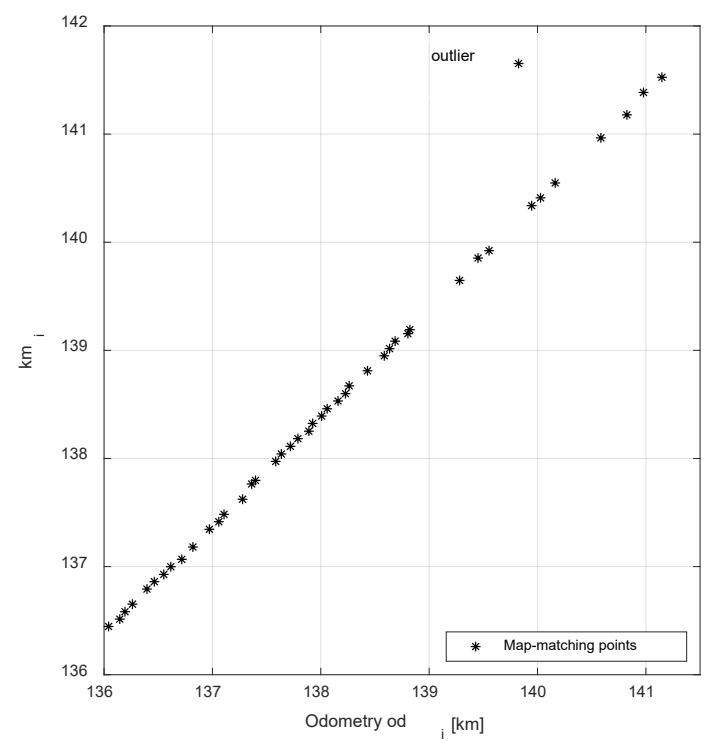

Figure 4: example of GPS acquisition with a standard GPS system adopted on railway vehicles.

Subsequent points $\mathrm{km}_{i}$ of the y-axis show a certain level of irregularity, and the presence of an outlier is clearly visible, due the wrong GPS acquisition already shown in Figure 1. This fact demonstrates that a further step must be carried out in the algorithm to exclude outliers and to obtain a more regular output. This second step should also allow to find a linear correlation between the odometry and the distance covered by the train estimated through the GPS signal, to be used to correct the odometry signal in the eventuality of drift occurrences.

The proposed solution consists in interpolating with a linear function the odometry acquisitions $\left(o_{i}\right)$ and the estimated positions $\left(k m_{i}\right)$. The slope $m$ and the intercept $q$ of the best fit line allow to define a function $\left(P K=m * o d_{i}+q\right)$ exploitable to calculate an estimated milestone position PK for each odometry acquisitions. The geo-localization algorithm can in such a way output an estimated PK for every odometry data (i.e. every $100 \mathrm{~ms}$ ), with a better time rate compared to the availability of GPS 
signal. The next section 3.2 demonstrates how this procedure can be more effectively carried out by a robust-fit method, also known as robust regression, rather than a least squares (LS) regression.

\subsection{Robust fit}

The linear regression can be performed when a suitable number of map-matched points $\left(\operatorname{od}_{i}, k m_{i}\right)$ is available. During the train run, the algorithm performs an automatic check to verify that the available number of map-matched points is greater than a predefined minimum $\left(p t_{\text {min }}\right)$, and that the distance covered by the train since the last robust fit has been evaluated is higher than a minimum value $\left(k m_{\min }\right)$. If these conditions are satisfied, a robust fit is executed.

The adoption of a robust fit procedure as an alternative to LS is justified by the fact that several studies have shown that the latter is vulnerable to the violation of the hypotheses that the noise corrupting the data has null mean value [21],[22]. For example, in the case in which the error distribution is asymmetric or affected by extreme outliers, even a single "bad" observation can completely perturb the LS estimate. To overcome this weakness, many robust techniques have been proposed [23], among which M-estimators, High Breakdown-Point and Bounded-Influence Estimators [24],[25] have been applied in mechanical engineering field.

In the following, the algorithm here employed for the robust fit is described, and the main differences with respect to the standard LS estimator are highlighted. Considering a linear model:

$$
y_{i}=b_{1} x_{i 1}+b_{2} x_{i 2}+\cdots+b_{m} x_{i m}+e_{i}=\boldsymbol{x}_{\boldsymbol{i}}^{\prime} \boldsymbol{b}+e_{i}
$$

the vector $\boldsymbol{b}=\left[b_{1}, \cdots, b_{m}\right]$ of the parameters to be estimated, is obtained in the standard LS algorithm by minimizing the normalized quadratic error:

$$
\sum_{i=1}^{n}\left(e_{i}\right)^{2}=\sum_{i=1}^{n}\left(y_{i}-x_{i}^{\prime} \boldsymbol{b}\right)^{2}
$$

The robust estimation, on the contrary, minimizes the sum of a suitable weighting function $\rho\left(e_{i}\right)$ of the normalized errors $e_{i}=\left(y_{i}-\boldsymbol{x}_{\boldsymbol{i}}^{\prime} \boldsymbol{b}\right)$ : 


$$
\sum_{i=1}^{n} \rho\left(e_{i}\right)=\sum_{i=1}^{n} \rho\left(y_{i}-\boldsymbol{x}_{\boldsymbol{i}}^{\prime} \boldsymbol{b}\right)
$$

This is obtained by putting equal to zero the derivative of eq. (3) with respect to $e_{i}$. The selection of the function $\rho\left(e_{i}\right)$ is the core of the robust regression and several approaches can be used. In this paper, the so-called M-estimators are employed. A full discussion is presents in [18] and only the algorithm is described hereafter.

The vector $\widehat{\boldsymbol{b}}$, which is the solution of the $m$ equations:

$$
\sum_{i=1}^{n} \psi\left(e_{i}\right) \frac{\partial e_{i}}{\partial b_{j}}=0, \quad \text { for } j=1 \ldots m
$$

is the M-estimator of $\boldsymbol{b}$, given the function $\rho\left(e_{i}\right)$. In statistical terms, the quantity

$$
\psi\left(e_{i}\right)=\frac{d \rho\left(e_{i}\right)}{d e_{i}}
$$

can be proven to be proportional to the influence function of $\rho\left(e_{i}\right)$, see [18].

In order to consider an estimator as a robust one, the influence of a single additional observation has not to determine a substantial error during the fitting. To implement this fact, a weight function $w\left(e_{i}\right)$ is introduced:

$$
w\left(e_{i}\right)=\frac{\psi\left(e_{i}\right)}{e_{i}}
$$

In this way, eq. (4) becomes:

$$
\sum_{i=1}^{n} w\left(e_{i}\right) e_{i} \frac{\partial e_{i}}{\partial b_{j}}=0, \quad \text { for } j=1 \ldots m
$$

The system of equations (7) correspond to the system which is obtained in the so called iterated re-weighted least squares (IRLS) problem, that is when the following minimization problem is solved:

$$
\min \sum_{i=1}^{n} w\left(e_{i}^{(t-1)}\right) e_{i}^{2}
$$


In eq. (8), the parameter $t$ is the index of the iteration number, while the weights $w\left(e_{i}^{(t-1)}\right)$ are calculated in each iteration.

\subsubsection{Implementation of the ILRS algorithm for robust fit using M-estimators}

The train position is finally estimated by using M-estimate, thus the following objective function has to be minimized:

$$
\sum_{i=1}^{n} \rho\left(e_{i}\right)=\sum_{i=1}^{n} \rho\left(\sum_{j=1}^{m}\left(\frac{k m_{i}-\boldsymbol{o d}_{\boldsymbol{j}} \boldsymbol{A}_{\boldsymbol{i}}^{(\boldsymbol{t})}}{\sigma}\right)\right)
$$

where the new parameter $\sigma$ here introduced is the scale estimate, i.e. a metrics to normalize the errors, which is necessary, since in this case physical quantities are considered instead of statistical ones.

The solution is obtained by minimizing eq. (9), that is $\sum w_{i} e_{i}^{2}$. Since the weights $w_{i}=w\left(e_{i}\right)$ are function of the errors $e_{i}$, the errors on their turn depend on the estimated coefficients, and the estimated coefficients on the weights. Therefore, the solution must be obtained by means of an iterative procedure, which explains the name of the algorithm.

The first step is the initial estimation of $\boldsymbol{A}^{(0)}$ and standard LS are used to obtain it. Then, in each iteration $t$, the errors $e_{i}^{(t-1)}$ are calculated (second step), along with their associated weights $w_{i}^{(t-1)}=$ $w\left[e_{i}^{(t-1)}\right]$ from the previous iteration, and a new weighted-least-squares estimation is obtained

$$
A^{(t)}=\left[X^{\prime} W^{(t-1)} X\right]^{-1} X^{\prime} W^{(t-1)}
$$

where $\boldsymbol{X}$ is the so-called model matrix, with $\operatorname{od}_{i}$ as its $\mathrm{i}^{\text {th }}$ row, and $W^{(t-1)}=\operatorname{diag}\left\{w_{i}^{(t-1)}\right\}$ is the current weight matrix (third step).

Second and third steps are iterated until convergence of the estimated coefficients is obtained. Despite the ILRS algorithm has a simple implementation, because it needs to iteratively solve a weighted LS problem, there are two critical points: the first is the selection of the error function $\rho\left(e_{i}\right)$, see next section, from which the weights $w\left(e_{i}\right)$ originate directly. The second is the stop criterion of the iterations. In this paper, the algorithm stops after 50 iterations unless convergency is reached.

\subsubsection{Selection of the weight function}


For standard LS, the error function, the influence function (up to a constant) and the weights are, respectively:

$$
\rho(e)=\frac{e^{2}}{2}, \quad \psi(e)=e, \quad w(e)=1
$$

In statistical terms, this estimator is convex, the influence function $\psi(e)$ is a straight line as a function of $e$, therefore $\psi(e)$ is not bounded and this estimator is not robust, as well know.

In this paper the Welsch's error function will be used. It has been introduced to reduce the effects of large errors and is defined as

$$
\rho(e)=\frac{c^{2}}{2}\left(1-\exp \left(-\left(\frac{|e|}{c}\right)^{2}\right)\right)
$$

thus, the influence function (up to a constant) and the weights are, respectively

$$
\begin{aligned}
& \psi(e)=e \exp \left(-\left(\frac{|e|}{c}\right)^{2}\right) \\
& w(e)=\exp \left(-\left(\frac{|e|}{c}\right)^{2}\right)
\end{aligned}
$$

The so-called tuning parameter $c$ for $95 \%$ asymptotic efficiency with respect to a normal distribution is $c=2.9846 \sigma$.

Figure 5 shows an example of the performances of the robust fit algorithm (continuous line) in comparison to least square regression (dashed line), highlighting the beneficial effects in case of lowquality GPS acquisition. The comparative analysis is shown on the results already described in Figure 4. As mentioned, the map-matching procedure is affected by a remarkable outlier, which is successfully neglected in the robust fit result, following at best the point distribution. On the contrary, the dashed line resulting from a least square regression is remarkably affected by the presence of the outlier. The figure legend also reports the slope values $m$ of the two fitting lines. In the case of the least square regression the difference in the $m$ is about $2.9 \%$, which would result in a relevant error. 


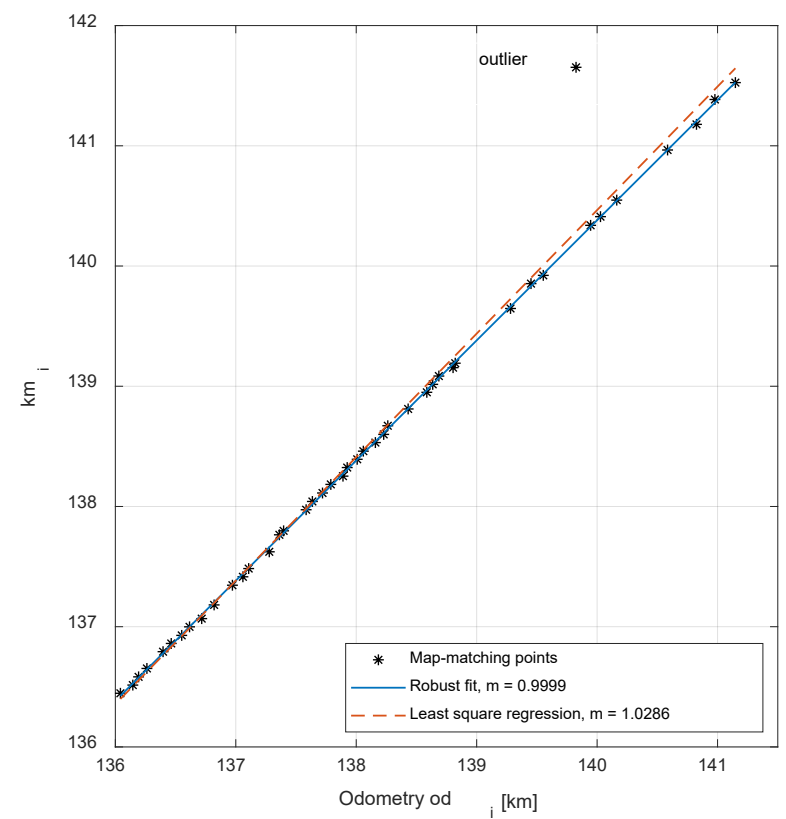

Figure 5: Comparison between Robust fit and linear regression results.

\subsection{Triggering the space-based windows for the RMS analysis of accelerations}

The procedure described in the previous paragraph requires the storage of a proper amount of data before that a robust fit analysis can be performed and before that the estimated PK values can be output based on the fitting line. Even if the algorithm can be run on-board train during the vehicle run, this procedure cannot be considered therefore as an actual real-time one. Nevertheless, thanks to this feature, acceleration and braking phases can be identified through a check on the speed profile, so that the robust fit analysis can be performed only on homogeneous data belonging to these transient phases, and potential drifts affecting the odometry can be compensated and corrected.

On the other hand, the need to trigger the start and end of the windows exploited for RMS analysis requires a continuous calculation of the milestone position, in real-time during the train run. A further thread of the algorithm fulfils the task.

During the train run, if at least a robust fit has been computed, the milestone position PK is continuously evaluated based on the last parameters $m$ and $q$ available. Figure 6 exemplifies this procedure through a set of $k m_{i}$ and $o d_{i}$ data gathered during experimental tests on a high-speed 
vehicle, indicated with star markers and referring to a line section of $8 \mathrm{~km}$. Let us focus on the range between km 191 and km 194 in Figure 6a (i.e. extrapolation points): based on the current odometry acquisitions, the PK values are estimated as an extrapolation from the previous robust fit line evaluated in the window $\mathrm{km}_{\min }$. An enlargement of the results is represented in Figure 6b: the estimated PK (square markers) are sufficiently accurate and close to the actual results generated from the on-going map-matching procedure (star markers). As an example, when comparing at odometry 191.045 (Figure 6b) the map-matched point and the projection from the previous robust fit, a $3.6 \mathrm{~m}$ estimation error is observed. It follows that the extrapolation of PK data from the previous robust fit can be fed in real time to the diagnostic boards to trigger the analysis of accelerations.

At the same time, the map-matching and robust fit tasks keep on running on a parallel threads: the algorithm parameter $k m_{r f}$ (see Figure 6c) defines the number of kilometres to be run before that a new robust fit is evaluated. A certain level of overlap is guaranteed between subsequent robust fit windows, to make the output of this extrapolation more stable. Figure $6 \mathrm{~d}$ reports an enlargement of this process, representing the data extrapolated from the $\mathrm{i}^{\text {th }}$ robust fit (square marker, same as in Figure $6 \mathrm{~b})$, and the $\mathrm{i}^{\text {th+1 }}$ robust fit line based on the new map-matched data. 

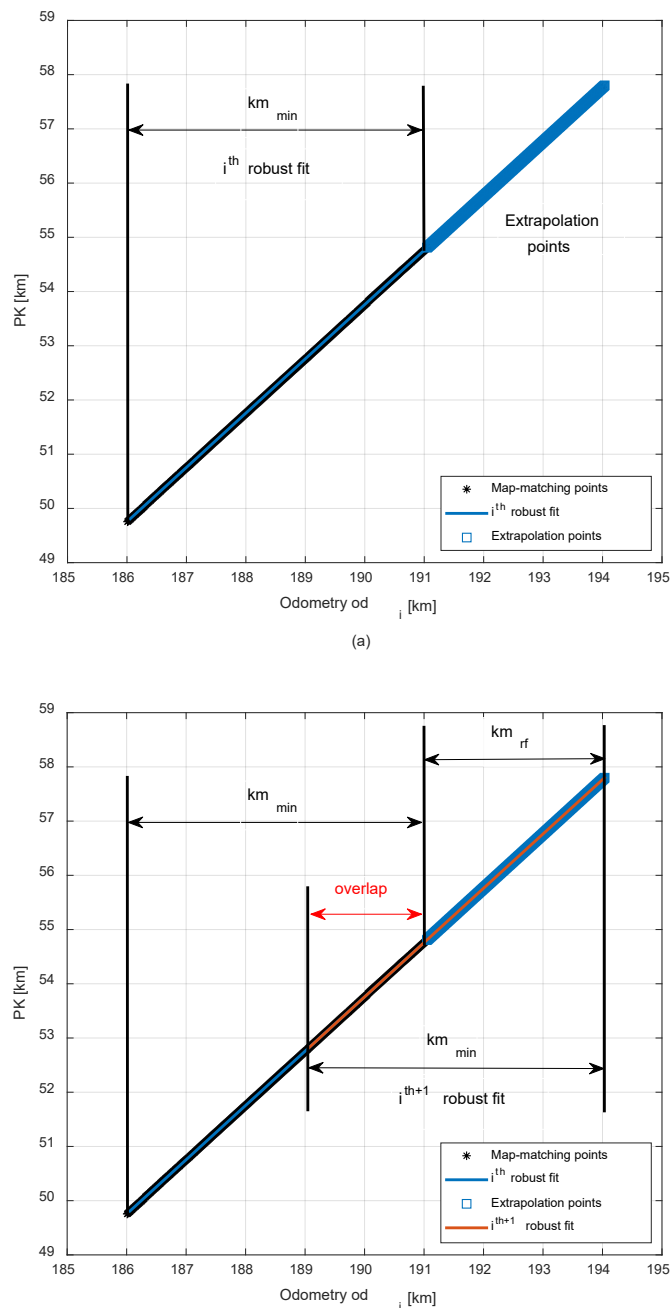
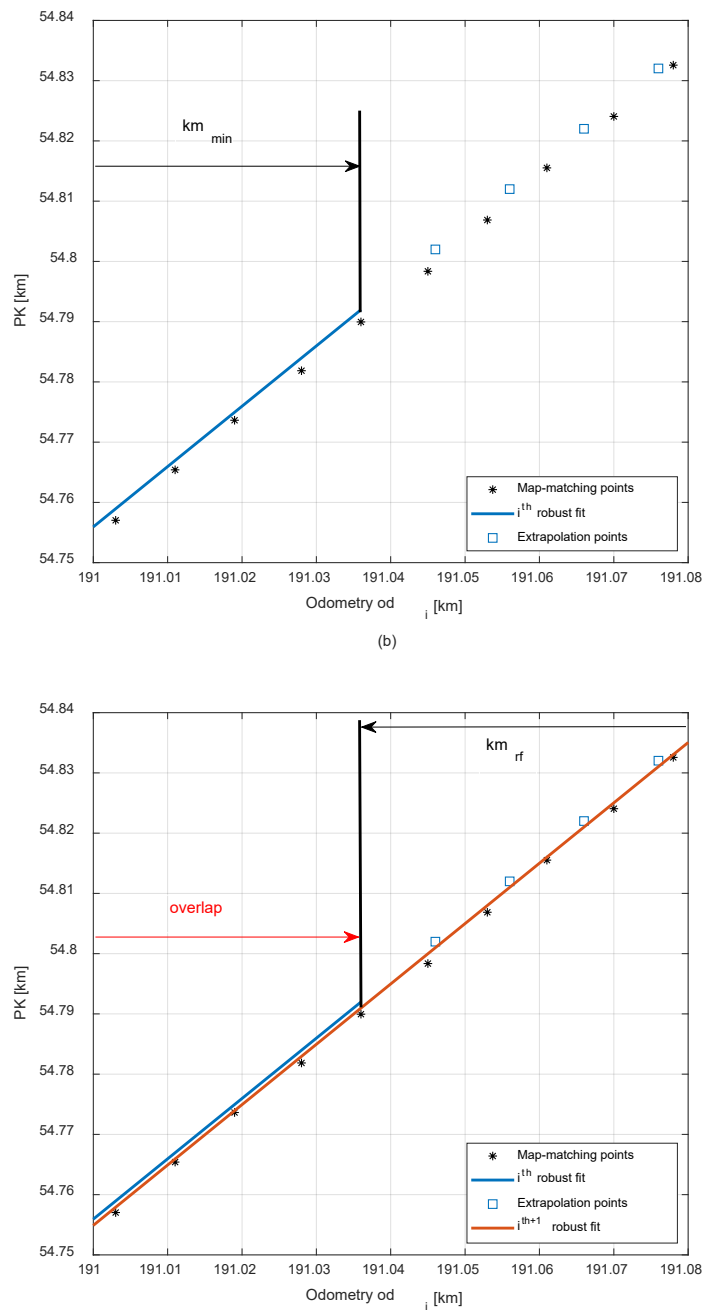

Figure 6: Extrapolation process to trigger acceleration analysis. a) Estimation of PK values based on previous robust-fit results. b) Enlargement of figure a. c) Evaluation of the subsequent robust-fit with window overlap. d) Enlargement of figure $\mathrm{c}$.

In any case, before performing a new robust fit after a distance $k m_{r f}$ has been furtherly run, a second check is performed to verify that the new $\mathrm{km}_{\min }$ window would include a number of map-matched points higher than a minimum value $p t_{\min }$. In such a way, in case the train enters a tunnel and GPS points are not available, the number of map-matched points do not satisfy the minimum $p t_{\min }$, and the milestone position PK keeps on being estimated based on the previous robust fit line.

The above described extrapolation allows to generate with an acceptable approximation the trigger signal which can drive the acceleration analysis in real time. The best values of the parameters $k m_{\min }$ and $\mathrm{km}_{r f}$ were set through a sensitivity analysis carried out off-line with different window 
lengths (from $2 \mathrm{~km}$ to $10 \mathrm{~km}$ ) and with different percentages of overlap. The final parameters are set to $k m_{\min }$ equal to $5 \mathrm{~km}$, and $\mathrm{km}_{r f}$ equal to $3 \mathrm{~km}$, leading to an overlap of $2 \mathrm{~km}$ between subsequent robust fit windows. Section 4 reports the results obtained during a test campaign on a high-speed train at $300 \mathrm{~km} / \mathrm{h}$.

\section{Results on a high-speed application}

The main aim of the experimental campaign is to test how the algorithm's output allows to generate diagnostic indicators as a function of the position where they are gathered, in a reliable and repetitive way [4]. The acquired data are automatically transmitted to a ground server. The first relevant result is the fact that RMS data originated from several repetition of train runs on the same railway line are perfectly overlying both in term of peak amplitudes and location along the line. Figure 7 reports the RMS data evaluated on vertical accelerations of the last bogie of the train, with window length 100 $m$ and frequency range $0-40 \mathrm{~Hz}$. The RMS correspond to data acquired during five train runs repeated along the same track with maximum speed equal to $300 \mathrm{~km} / \mathrm{h}$. For an easy-of-readiness of the figure, the reported analysis corresponds to a track section of $14 \mathrm{~km}$, even if similar results are obtained along the entire railway line tested (around $200 \mathrm{~km}$ ) and on other lines too. Similar results are also obtained for the other bogies of the train, all instrumented.
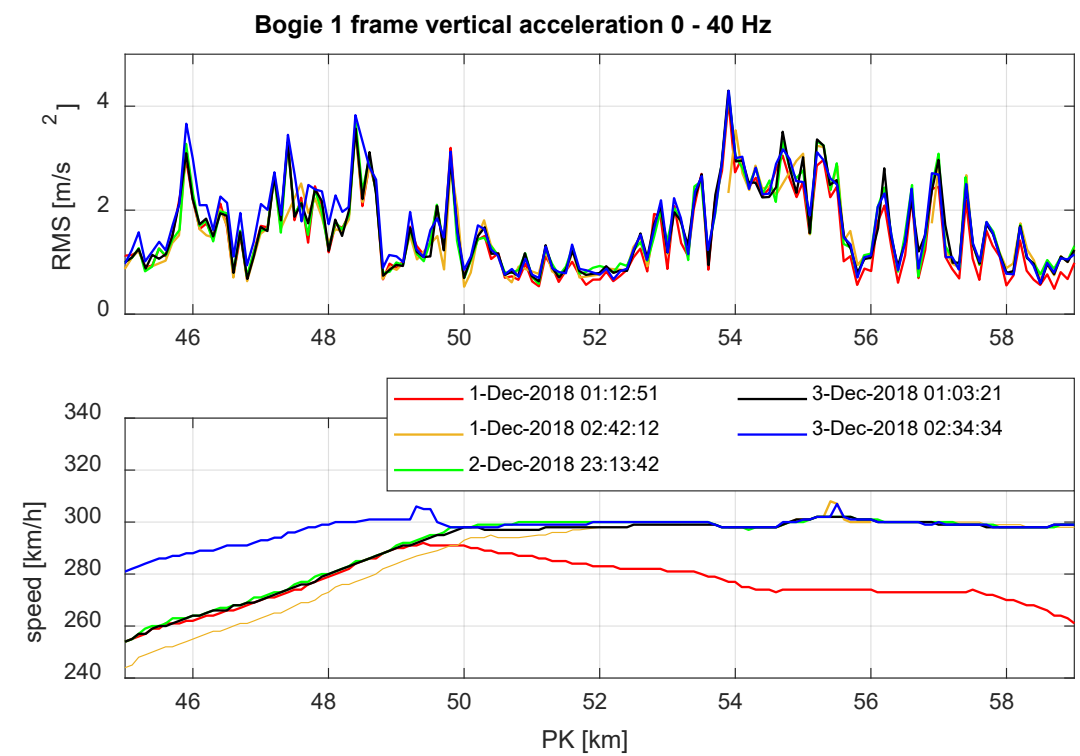

Figure 7: Repeatability of RMS bogie accelerations; accelerometer installed in the middle of the bogie frame. 
An excellent overlap of the RMS histories is obtained for all the test runs. When the train speeds are reasonably similar (e.g. in the range $260-300 \mathrm{~km} / \mathrm{h}$ ) also the RMS peak values are comparable, enabling the possibility to perform a trend analysis on data aggregated over a speed range admitting sensible variations from the nominal commercial speed.

A second positive result is related to the consistency of RMS signal obtained from different bogies of the train. Since the positioning output of the geo-localization algorithm corresponds to the actual position where the GPS antenna is placed (i.e. leading or trailing coach), the data generated by the different diagnostic boards placed along the train need to be compensated, according to the actual distance of the instrumented pantograph or bogie from the GPS antenna. Figure 8 reports the results corresponding to the RMS of accelerations measured on the leading bogie of the leading, trailing and middle coaches before carrying out the distance compensation. Since the train is $200 \mathrm{~m}$ long, the same RMS peak is detected with a shift of $100 \mathrm{~m}$ in the signals corresponding to the leading and the middle coaches, and with a shift of $200 \mathrm{~m}$ in the signals related to the leading and the trailing coaches. Information given by all the bogies along the train shows a positive consistence.

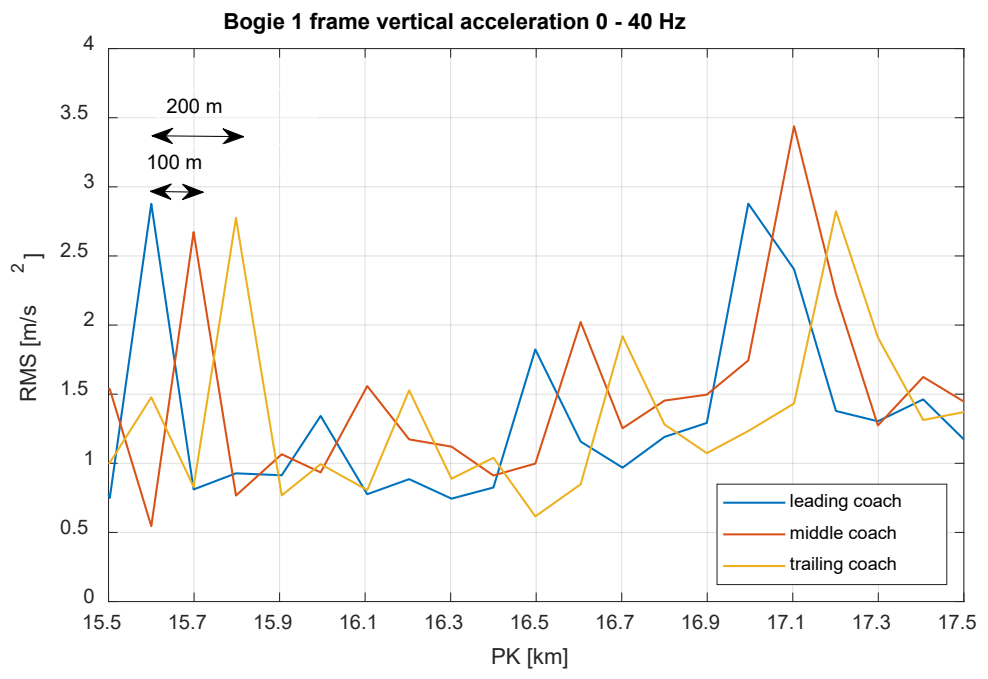

Figure 8: RMS of bogie acceleration in the 0-40 Hz range. Leading bogie of leading, middle and trailing coaches. RMS evaluated over a $100 \mathrm{~m}$ window.

The same repeatability is confirmed by the diagnostic data related to pantograph sensors. In this case two algorithms are run, as comprehensively described in [5],[26]. Figure 9 represent the repeatability 
of the RMS data evaluated through the first algorithm, computing RMS over a long space-based window (length $1 \mathrm{~km}$ ) and for different frequency bands distinctive of the dynamic interaction between the pantograph and the overhead contact line. Some exemplary results are reported, related to the frequency bands of 5-30 Hz (Figure 9a) and 20-110 Hz (Figure 9b). For each one of the two figures, the first two subplots (RMS1 and RMS2) report the values corresponding to the accelerations of the two collectors of the pantograph [5]. The bottom subplot represents the train speed. Two train runs have been selected, being the ones in which the speed profile is more regular and closer to the maximum speed of $300 \mathrm{~km} / \mathrm{h}$. The reported data overlay in a very satisfactorily way.
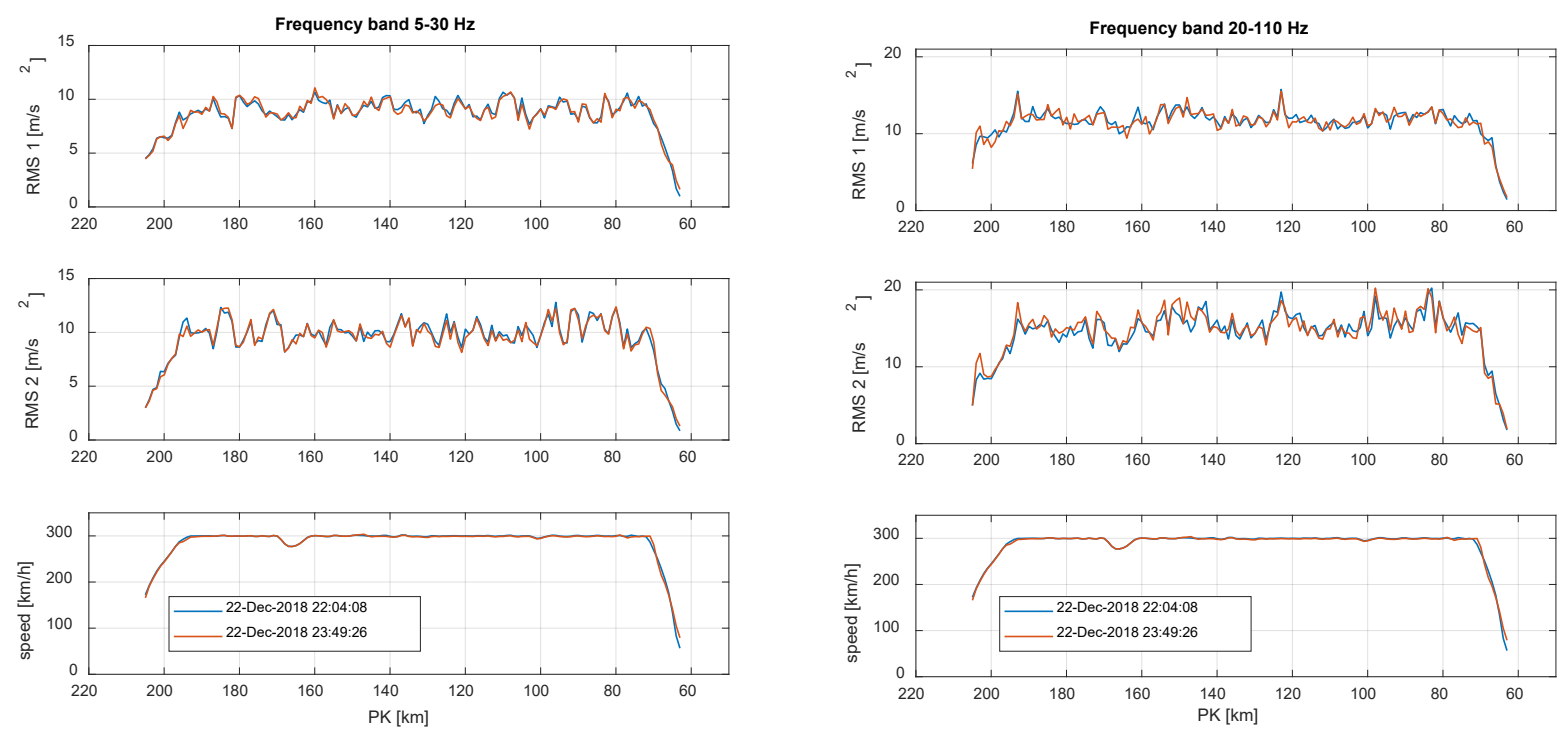

Figure 9: RMS evaluated with window length $1 \mathrm{~km}$. a) Frequency band 5-30 Hz. b) Frequency band 20-110 Hz.

Finally, Figure 10 shows the results generated by the second algorithm, evaluating the RMS on a short, time-based window with duration $\mathrm{T}=0.2 \mathrm{~s}[5]$ and $90 \%$ of window overlap (frequency content $0.5-250 \mathrm{~Hz}$ ). Once again, the superposition of data coming from different runs allow to identify the RMS peaks at the same location. This second algorithm is aimed at identifying local defects in the overhead contact line, and it can be considered even a more stringent validation of the accuracy of the system, the RMS window being rather narrow (i.e. $\mathrm{T}=0.2 \mathrm{~s}$ ). 

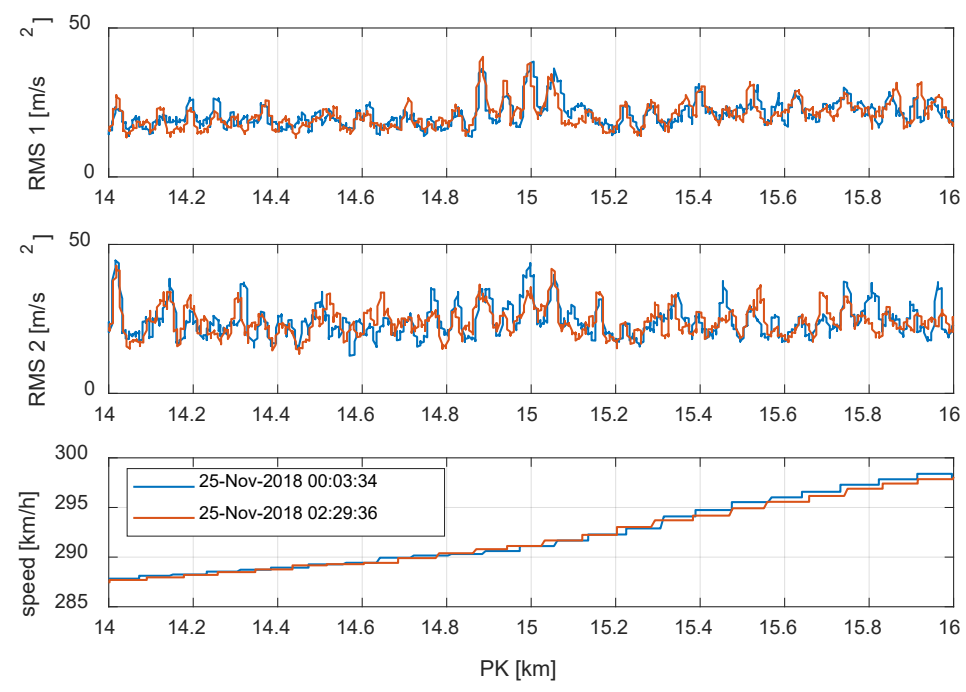

Figure 10: RMS of pantograph acceleration over a short time window ( $T=0.2 \mathrm{~s})$. Repetition of two test runs.

\section{Conclusions}

The paper describes a solution for a train geo-localization system adoptable in a rolling-stock based diagnostic system. The aim of the work is to provide an instrument to associate any diagnostic indicator elaborated in real time during the train run to the actual position along the line where its source data have been acquired. The possibility to collect data histories which can be automatically overlapped and compared poses the bases for an automatic trend analysis of data gathered from a commercial train fleet.

The proposed algorithm is based on a map-matching procedure carried out on GPS acquisitions, followed by a robust fit technique to associate the odometry acquisitions to the actual milestone positions along the line. The procedure permits to discard outliers in GPS acquisitions, to provide a regular output of the positioning signal also in the presence of tunnels, and to compensate for drift in the odometry signal. When performed in real-time, it can be also be exploited to trigger the analysis of the raw data, so that the diagnostic indexes generated by all the trains of an instrumented fleet correspond to the same track sections.

Results from the tests on a high-speed train showed a good repeatability both in terms of RMS values and of their positioning, posing the basis for a further step in the development of condition-based maintenance techniques in railways. 


\section{Acknowledgments}

This work is part of the CBM project of the Joint Research Center, established by Fondazione Politecnico di Milano.

The authors would like to thank Dr. Filippo Cangioli for developing the Matlab code during his Ph.D. studies.

\section{References}

[1] Chunsheng Li, Shihui Luo, Colin Cole \& Maksym Spiryagin (2017) An overview: modern techniques for railway vehicle on-board health monitoring systems, Vehicle System Dynamics, 55:7,1045-1070, DOI:10.1080/00423114.2017.1296963

[2] Ward, C. P., Weston, P. F., Stewart, E. J. C., Li, H., Goodall, R. M., Roberts, C., ... Dixon, R. (2011). Condition Monitoring Opportunities Using Vehicle-Based Sensors. Proceedings of the Institution of Mechanical Engineers, Part F: Journal of Rail and Rapid Transit, 225(2), 202-218. https://doi.org/10.1177/09544097JRRT406

[3] Cangioli, F.; Carnevale, M.; Chatterton, S.; De Rosa, A.; Mazzola, L. Experimental results on condition monitoring of railway infrastructure and rolling stock. In Proceedings of the First World Congress on Condition Monitoring 2017 (WCCM 2017), London, UK, 13-16 June 2017.

[4] Diana G.; Firmi P.; Caposciutti M.; Zocco M.A.; Gherardi F. Condition Based Maintenance in railway asset: use of the commercial fleet as a data probe. Proceedings of the 12th World Congress on Railway Research (WCRR19). October 28 to November 1, 2019, Tokyo, Japan.

[5] Carnevale M., Collina A.: Processing of collector acceleration data for condition-based monitoring of overhead lines. Proceedings of the Institution of Mechanical Engineers, Part F: Journal of Rail and Rapid Transit (2014): DOI:https://doi.org/10.1177/0954409714545637.

[6] Carnevale M., Collina A., Facchinetti A. and Gasparetto L.: Overhead Line Diagnostic System for High Speed Pantographs. The Second International Conference on Railway Technology: Research, Development and Maintenance, Ajaccio, Corsica, France 8-11 April 2014. DOI: 10.4203/ccp.104.140. 
[7] Gerlach, K., Zu Hörste, M.M. A precise digital map for GALILEO-based train positioning systems. (2009) 9th International Conference on Intelligent Transport Systems Telecommunications, ITST 2009, art. no. 5399330, pp. 343-347. DOI: 10.1109/ITST.2009.5399330

[8] Xi-Hui, Y., Jian, W., Bai-Gen, C., Wei, S. A novel automatic track identification algorithm based on LTS-Hausdorff distance (2011) IEEE Conference on Intelligent Transportation Systems, Proceedings, ITSC, art. no. 6082896, pp. 1984-1988. DOI: 10.1109/ITSC.2011.6082896.

[9] Saab, S.S. A map matching approach for train positioning part I: Development and analysis (2000) IEEE Transactions on Vehicular Technology, 49 (2), pp. 467-475. DOI: 10.1109/25.832978

[10] Saab, S.S. A map matching approach for train positioning part II: Application and experimentation (2000) IEEE Transactions on Vehicular Technology, 49 (2), pp. 476-484. DOI: 10.1109/25.832979

[11] Gerlach, K., Rahmig, C. Multi-hypothesis based map-matching algorithm for precise train positioning (2009) 12th International Conference on Information Fusion, FUSION 2009, art. no. 5203750, pp. 1363-1369.

[12] Lüddecke, K., Rahmig, C. Evaluating multiple GNSS data in a multi-hypothesis based map-matching algorithm for train positioning (2011) IEEE Intelligent Vehicles Symposium, Proceedings, art. no. 5940494, pp. 1037-1042. DOI: 10.1109/IVS.2011.5940494

[13] B. Allotta, P. D'Adamio, M. Malvezzi, L. Pugi, A. Ridolfi and G. Vettori, A localization algorithm for railway vehicles, 2015 IEEE International Instrumentation and Measurement Technology Conference (I2MTC) Proceedings, Pisa, 2015, pp. 681-686, doi: 10.1109/I2MTC.2015.7151350.

[14] Tsunashima H, Naganuma Y, Matsumoto A, Mizuma T, Mori H. Japanese railway condition monitoring of tracks using in-service vehicle. 5th IET Conference on Railway Condition Monitoring and NonDestructive Testing (RCM 2011), Derby, 2011, pp. 1-6. doi: 10.1049/cp.2011.0587

[15] Tsunashima H, Naganuma Y, Matsumoto A, Mizuma T, Mori H. Condition Monitoring of Railway Track Using In-Service Vehicle. In Perpinya X, editor. Reliability and Safety in Railway. InTech; 2012. DOI: $10.5772 / 35205$.

[16] Tsunashima H, Naganuma Y, Kobayashi T. (2014) Track geometry estimation from car-body vibration, Vehicle System Dynamics, 52:sup1, 207-219, DOI: 10.1080/00423114.2014.889836

[17] Ackroyd, P., Angelo, S., Nejikovsky, B., Stevens, J. Remote ride quality monitoring of acela train set performance (2002) Proceedings of the IEEE/ASME Joint Railroad Conference, pp. 171-178. 
[18] Pennacchi P., Robust estimate of excitations in mechanical systems using M-estimators - Theoretical background, Journal of Sound and Vibration, Vol. 310, No. 4-5, 2008, ISSN 0022-460X, pp. 923-946, DOI information: $10.1016 /$ j.jsv.2007.08.007.

[19] P. Weston, C. Roberts, G. Yeo \& E. Stewart (2015) Perspectives on railway track geometry condition monitoring from in-service railway vehicles, Vehicle System Dynamics, 53:7, 1063-1091, DOI: 10.1080/00423114.2015.1034730.

[20] European Global Navigation Satellite Systems Agency (GSA), European Geostationary Navigation Overlay Service (EGNOS), https://www.gsa.europa.eu/european-gnss/egnos/services/performance-overview, webpage, date accessed 2020/09/04.

[21] Pennacchi P., Robust estimation of excitations in mechanical systems using M-estimators Experimental applications, Journal of Sound and Vibration, Vol. 319, No. 1-2, 2008, ISSN 0022-460X, pp. 140-162, DOI information: 10.1016/j.jsv.2008.05.017.

[22] Pennacchi P., Vania A., Bachschmid N., Increasing the robustness of fault identification in rotor dynamics by means of M-estimators, Mechanical Systems and Signal Processing, Vol. 21, No. 8, 2007, ISSN 0888-3270, pp. 3003-3029.

[23] Bickel P., 1983, "Robust Estimation”, in Encyclopedia of Statistical Sciences, Eds. S. Kotz, N.L. Johnson, C.B. Read, N. Balakrishnan and B. Vidakovic, Vol. 8, John Wiley \& Sons, Ltd, 157-163.

[24] Pennacchi P., Chatterton S., Ricci R., Rotor Balancing Using High Breakdown-Point and BoundedInfluence Estimators, Mechanical Systems and Signal Processing, Vol. 24, No. 3, 2010, ISSN 0888-3270, pp. 860-872, DOI information:10.1016/j.ymssp.2009.10.004.

[25] Chatterton S., Ricci R., Pennacchi P., Application and Comparison of High Breakdown-Point and Bounded-Influence Estimators to Rotor Balancing, Transactions of ASME - Journal of Vibration and Acoustics, Vol. 132, No. 6, 2010, ISSN 1048-9002, pp. 064502-1-064502-6, DOI information: $10.1115 / 1.4001842$.

[26] Carnevale, M., Collina, A., Palmiotto, M. Condition monitoring of railway overhead lines: Correlation between geometrical parameters and performance parameters (2017) WCCM 2017 - 1st World Congress on Condition Monitoring 2017. 